\title{
Kernos
}

Revue internationale et pluridisciplinaire de religion grecque antique

$27 \mid 2014$

Varia

\section{Caitlín E. BARRETT, Egyptianizing Figurines from Delos}

\section{Richard Veymiers}

\section{(2) OpenEdition}

\section{Journals}

Édition électronique

URL : http://journals.openedition.org/kernos/2254

DOI : 10.4000/kernos.2254

ISSN : 2034-7871

Éditeur

Centre international d'étude de la religion grecque antique

Édition imprimée

Date de publication : 1 novembre 2014

Pagination : 484-490

ISBN : 978-2-87562-055-2

ISSN : 0776-3824

\section{Référence électronique}

Richard Veymiers, «Caitlín E. Barrett, Egyptianizing Figurines from Delos », Kernos [En ligne], 27 | 2014,

mis en ligne le 12 novembre 2014, consulté le 22 septembre 2020. URL : http://

journals.openedition.org/kernos/2254; DOI : https://doi.org/10.4000/kernos.2254

Ce document a été généré automatiquement le 22 septembre 2020.

Kernos 


\title{
Caitlín E. BARRETT, Egyptianizing Figurines from Delos
}

\author{
Richard Veymiers
}

\section{RÉFÉRENCE}

Caitlín E. BARRETT, Egyptianizing Figurines from Delos. A Study in Hellenistic Religion, Leiden/ Boston, Brill, 2011. 1 vol. 16,5 × 24,5 cm, 731 p. + pl. (Columbia Studies in the Classical Tradition, vol. 36). ISBN : 978-90-04-20797-4.

1 Issue d'une thèse de doctorat soutenue en 2009 à l'Université de Yale, l'importante monographie que nous offre C.E. Barrett révèle tout l'intérêt de la culture matérielle, et en particulier de l'univers coroplathique, pour affiner notre connaissance de la lived religion à l'époque hellénistique, au-delà du concept monolithique de religion « domestique », de la dichotomie artificielle entre sphères « populaire » et « officielle ». Exceptionnel conservatoire archéologique, l'île de Délos a livré de très nombreux vases plastiques et figurines en terre cuite, dont près de 1500 exemplaires ont été jusqu'à présent publiés par A. Laumonier, A. Bovon et P. Hatzidakis ${ }^{1}$. Ce sont 82 monuments soit environ $5 \%$ du corpus - identifiés par l'A. comme égyptiens ou surtout égyptisants qui constituent le cœur matériel de cette enquête interdisciplinaire. Épousant une séduisante perspective anthropologique, l'A. cherche à réintégrer ces artefacts dans leur réseau de relations sociales - ce qu'on appelle par ailleurs l'agency - pour mieux comprendre les messages qu'ils véhiculent et les pratiques qu'ils recouvrent dans leurs contextes respectifs.

2 Cette étude qui vise à une meilleure compréhension de la société délienne et de son paysage religieux est précédée d'une introduction programmatique, faisant office de chapitre 1 (Egyptianizing Cult at a Crossroads of Hellenistic Trade), où il aurait été bienvenu de définir clairement ce qu'on entendait par "egyptianizing ${ }^{2}$, puisque l'étiquette s'applique également ici aux sanctuaires isiaques et aux cultes qu'ils abritent. Si l'influence des Ptolémées se manifeste à Délos dès le protectorat accordé en 287 à la 
Ligue des Nésiotes, qui y instaure leur culte royal, les divinités «égyptisantes » font parallèlement - et indépendamment - leur apparition sur l'île à l'initiative d'un prêtre memphite, selon la célèbre "chronique $»^{3}$ du plus ancien des trois Sarapieia $a^{4}$. Parmi la multiplicité des traditions religieuses égyptiennes, rappelée à juste titre par l'A., ce sont celles d'Alexandrie et de Basse-Égypte qui semblent émerger de ces terres cuites datant pour la plupart de la fin du $\mathrm{II}^{\mathrm{e}}$ ou du début du $\mathrm{I}^{\mathrm{er}} \mathrm{s}$. av. J.-C. Sans doute aurait-il été utile, pour les lecteurs non familiers avec les travaux de P. Roussel ou M.-Fr. Baslez entre autres ${ }^{5}$, d'inclure dans cette introduction un développement historique sur les cultes isiaques à Délos et leurs autres manifestations matérielles, afin d'éprouver davantage la pertinence de ces concepts d'« hybridité » et de «syncrétisme» si souvent sollicités au cours de l'ouvrage.

3 Les terres cuites égyptiennes et égyptisantes de Délos se prêtent à divers niveaux d'analyse, définissant autant de chapitres, une perspective totale conforme à l'approche anthropologique générale. L'A. examine avec grande minutie la composition des matériaux, les techniques de fabrication, le contenu iconographique et le contexte archéologique des monuments, passant ainsi du point de vue des producteurs à celui des consommateurs. Complément indispensable à l'analyse, un catalogue (Annexe B) regroupe toutes les données disponibles pour les artefacts ${ }^{6}$, en les classant selon l'ordre du numéro d'inventaire des musées archéologiques de Délos et de Mykonos. La plupart des monuments font l'objet de deux supports visuels réalisés par l'A.: un dessin agrémentant la fiche d'enregistrement et une photographie intégrée, avec une numérotation propre (D), dans des planches en couleur ou noir et blanc organisées thématiquement à la fin du volume - et qui auraient pu être aussi numérotées pour plus de commodité.

4 Afin de définir le cadre de production, c'est-à-dire l'origine géographique des terres cuites, et la tradition technologique dans laquelle s'inscrivaient leurs artisans, l'A. s'est attachée de manière très pertinente à comparer leurs caractéristiques matérielles et techniques à celles des figurines déliennes montrant des thèmes helléniques, mais aussi à celles d'autres répertoires coroplathiques soigneusement choisis. Ce sont les terres cuites du Musée du Caire, dont Fr. Dunand ${ }^{7}$ avait étudié un échantillonnage, et celles du Musée de l'Agora d'Athènes, jadis publiées par D.B. Thompson ${ }^{8}$, soit deux corpus authentiquement égyptien et grec, qui lui offrent les comparanda nécessaires. La démarche est à nouveau directe, puisque l'A. les a examinées de visu, parfois de tactu, nous fournissant les photographies noir et blanc de 75 et 96 exemplaires numérotés ( $C$ et A) dans le recueil de planches. Non autorisée à procéder à une analyse pétrographique ou chimique des terres cuites, elle fait dans le chapitre 2 (Fabric Analysis: Evidence for the Local Origins of the Majority of Egyptianizing Figurines on Delos) une description macroscopique des pâtes utilisées, tenant compte d'une série de critères, ce qui lui permet de distinguer plusieurs groupes dans chaque répertoire (tableaux 1-10). Cette comparaison visuelle lui permet de conclure à la production locale de la majorité des terres cuites déliennes, quelle que soit leur iconographie. On trouve diverses importations, dont trois égyptiennes, mais la plupart - soit au moins 65 réalisations égyptisantes - utilisent une pâte fine et rougeâtre intégrant une argile cycladique. Le chapitre 3 (Manufacturing Techniques, Technological Style, and the Question of Egyptian Coroplasts on Delos) examine à travers chaque corpus le «style technologique » des terres cuites, que détermine l'ensemble des choix faits consciemment ou non par les artisans au cours des grandes étapes marquant le processus de fabrication (tableaux 11-15). Destiné à un marché local, l'artisanat coroplathique délien se rattache pour 
l'essentiel aux traditions technologiques grecques, mettant l'accent sur la qualité esthétique des œuvres, alors que les terres cuites égyptiennes, produites plus rapidement à certaines occasions, festives notamment, sont moins soignées, accordant davantage d'importance à leur valeur religieuse.

Les terres cuites égyptiennes et égyptisantes de Délos présentent divers types iconographiques que l'A. analyse longuement au sein du chapitre 4 (The Eye of the Sun and the Inundation of the Nile: Iconographic Evidence for Egyptian Theology on Delos) en les rapportant au système théologique égyptien à grand renfort de parallèles textuels et figuratifs d'époques pharaonique et gréco-romaine. Toutes ces images « religieuses » se réfèreraient, selon elle, à une thématique commune: l'arrière-plan mythique des festivités célébrant l'Inondation du Nil et la Nouvelle Année. L'arrivée de la crue était mise en relation par les Égyptiens avec le retour depuis la Nubie de la déesse lointaine incarnant l'œil solaire, à laquelle ils identifiaient Hathor, puis Isis, célébrée à Dendara en tant que «maîtresse du début de l'année ». Au moins quarante-trois terres cuites sont ainsi considérées comme diverses manifestations de l'œil du Soleil : Isis ou reine lagide en Isis ; les soi-disant " Aphrodite orientale»; les chiens sothiaques; Arsinoé II et sa double corne d'abondance. Six autres figurines présentent un caractère sexuel, sinon érotique, qui ferait allusion au hieros gamos de la déesse lointaine au moment de l'inondation: Harpocrate ithyphallique; les soi-disant «Baubo"; le couple Agathos Daimon(-Shaï) et Agathè Tychè(-Renenoutet). Le fruit de cette union sacrée est un enfant solaire, assimilé au roi régnant, auquel se réfèreraient quatorze terres cuites montrant divers types d'Harpocrate, dont l'un assis dans un lotus. Une douzaine de figurines ou vases plastiques représentent des Nubiens au faciès caractéristique, certains dansant ou chantant, qui seraient autant de membres de l'entourage de la déesse lointaine. Diverses formes de Bès et de Ptah-Patèque figureraient sur quelques terres cuites en raison des liens qui unissaient ces dieux-nains à la naissance de l'enfant solaire. La prédominance des couronnes florales, les multiples allusions au vin et à l'eau dans ce corpus coroplathique semblent confirmer sa relation avec le monde religieux des fêtes. L'effigie d'un Nubien à demi-allongé avec lanterne et amphore pourrait ainsi évoquer les beuveries nocturnes célébrant le retour de l'œil solaire lors de l'inondation, de même que celle d'un masque de Dionysos-Botrys composé de grappes de raisin. Enfin, deux piliers hermaïques avec caducée et pschent reproduisent une forme égyptisante d'Hermès, équivalent à Thot, qui avait convaincu la déesse éloignée de rentrer en Égypte ${ }^{9}$. La richesse théologique de toute cette imagerie conduit l'A. à suggérer que les producteurs des terres cuites déliennes avaient une connaissance très fine de la religion égyptienne, tout comme certains de leurs utilisateurs. Les coroplathes auraient même été capables d'innover, de créer de nouveaux types, tel l'Hermès-Thot, en combinant divers symboles pour traduire certains concepts égyptiens dans un langage visuel compréhensible par le public délien. L'expression de cette théologie nilotique à travers les terres cuites reflèterait l'existence de festivités locales réinterprétant ce qui se faisait en Égypte. L'équivalence entre le Nil et l'Inopos établie par Callimaque dans son Hymne à Délos (v. 206-208) en serait un autre écho, de même que les structures ${ }^{10}$ hydrauliques identifiées dans les Sarapieia de l'île.

Stimulante et ingénieuse à maints égards, cette analyse iconographique n'emporte toutefois pas totalement la conviction. L'A. y reconstitue un système cohérent (tableau 16) qui présente surtout l'avantage de donner un sens commun à toutes les images. Cette recherche sémantique qui sous-tend la constitution de ce véritable modèle interprétatif ne risque-t-elle pas néanmoins de nous éloigner de la réalité antique ? La 
volonté de tout rapporter à une grille de lecture globale semble en effet conduire l'A. à forcer à plusieurs reprises l'interprétation des monuments qu'elle a sous les yeux, sans approfondir d'autres pistes possibles. Est-il nécessaire d'évoquer les serpents Shaï et Renenoutet pour une statuette fragmentaire à l'effigie d'une dyade à la corne d'abondance (D53) qui a pu correspondre au couple anthropomorphe grec Agathos Daimon et Agathè Tychè, tel qu'il figure sur d'autres médias ${ }^{11}$ ? Il parait tout aussi audacieux d'identifier des terres cuites reproduisant des chiens assez communs (D3640) à des images allégoriques de l'étoile Sothis ou de considérer comme égyptisant un type de masque dionysiaque (D81) vraisemblablement originaire d'Amisos dans le Pont. Autrement dit, l'analyse iconographique souffre d'une approche quelque peu égyptocentrique. Il est certes éclairant de rechercher la signification originelle des thèmes égyptiens ou égyptisants véhiculés par ces terres cuites, mais peut-on l'appliquer telle quelle au milieu délien d'époque tardo-hellénistique, sans tenir compte des multiples filtres et relais par lesquels ont dû passer ces images? L'appropriation de référents égyptiens au sein d'un autre contexte culturel est loin d'être un processus linéaire, direct entre core et periphery ${ }^{12}$. Ces éléments sont bien souvent resémantisés pour véhiculer de nouvelles valeurs, lesquelles n'étaient pas forcément toujours religieuses. Certains des types affichés par ces terres cuites sont nettement en rapport avec l'univers isiaque, qui avait bel et bien exporté hors d'Égypte une forme de théologie nilotique osirienne, y compris à Délos comme l'atteste entre autres l'Hydreion du Sarapieion $\mathrm{C}^{13}$. D'autres pourraient renvoyer à une sphère plus culturelle, à l'instar de ces scènes "nilotiques » auxquelles l'A. consacre un petit excursus, mais qui auraient sans doute mérité davantage, puisqu'elle posent des problèmes d'analyse similaires. Ainsi que l'a révélé M.J. Versluys ${ }^{14}$, ces paysages du Nil en crue figurés le plus souvent sur des mosaïques et des peintures issues d'un environnement privé peuvent se prêter à un large spectre d'interprétations. Aucune de ces scènes n'a toutefois jusqu'ici été mise au jour à Délos ${ }^{15}$, où l'on rencontre en revanche ces terres cuites qui en seraient en quelque sorte les « équivalents tridimensionnels».

7 Centré sur les contextes de découverte, le chapitre 5 (A Contextual Analysis of the Findspots of Egyptian and Egyptianizing Terracotta Figurines on Delos) nous entraîne du côté des utilisateurs qui, comme le note justement l'A. malgré son postulat général, pouvaient percevoir diversement les terres cuites en fonction de leurs besoins, y reconnaissant par exemple des allusions politiques. Les publications antérieures étant généralement vagues sur les lieux de trouvaille, l'A. a procédé à une courageuse enquête archivistique à travers les carnets de fouille de l'École française d'Athènes (Annexe C), ce qui lui permit de repréciser le contexte de quarante-six objets. Il s'avère qu'une bonne moitié provient d'habitations et de rues implantées dans divers quartiers résidentiels de l'île, une large distribution indiquant qu'ils n'étaient pas propres à une communauté en particulier. Les maisons qui en ont fournis sont de toutes tailles, appartenant donc à des individus de divers statuts socio-économiques. Même pour celles dont on connaîtrait les propriétaires, telle la Maison de l'Hermès liée à la famille des Paconii, il est toutefois difficile, sinon impossible, d'identifier qui utilisait réellement ces artefacts. Cinq terres cuites ont été découvertes dans les ateliers coroplathiques du Quartier du Théâtre et de l'Agora des Italiens, détruits lors de l'un des sacs de lîle, tandis que quatre autres sont issues de boutiques commercialisant diverses catégories de produits. La nécropole de Rhénée a livré deux pièces importées d'Égypte dont le contexte exact demeure imprécis. Enfin, onze terres cuites sont originaires de sanctuaires authentiquement déliens, tels le hieron d'Apollon et l' 
Archegesion, mais aussi d'autres lieux de culte comme le naiskos de Dionysos, le Kabirion/ Samothrakeion et la Synagogue. Quant aux Sarapieia A et C, ils ont livré diverses figurines, mais aucune de type égyptisant, ce qui révèle combien ce genre de matériel est généralement peu fiable pour étayer l'attribution d'un sanctuaire.

Les terres cuites égyptisantes de Délos sont donc en rapport avec un large éventail de contextes (tableau 17) où elles avaient eu, à en croire l'A., essentiellement des fonctions religieuses. Certaines ont ainsi très bien pu être utilisées dans le cadre de rituels domestiques en prenant place dans les niches murales de demeures déliennes. Le postulat de lecture iconographique a toutefois conduit l'A. à considérer un peu abusivement que tous les utilisateurs de ces objets étaient des fidèles des cultes " égyptisants ». Tel a peut-être été le cas du ou des défunt(s) inhumé(s) avec des figurines d'Harpocrate ithyphallique, une iconographie véhiculant parfois une idée de renaissance ${ }^{16}$. Peut-on toutefois attribuer pour autant un symbolisme égyptien implicite au reste du mobilier de leur tombe alors qu'il n'a visiblement rien à voir avec l'Égypte ? Et supposer ainsi par exemple que des vases plastiques campaniens à l'effigie d'un poisson et d'un dauphin puissent renvoyer au culte délien d'Isis Pelagia - si tant est qu'il ait existé ? Faisant partie intégrante de la culture matérielle délienne tardohellénistique, les monuments égyptiens et égyptisants apparaissaient naturellement dans des ensembles diversifiés et hétérogènes. C'est ainsi que les deux ateliers repérés pouvaient produire conjointement des terres cuites de types grecs, égyptisants et/ou anatoliens. Toutes les associations n'étaient évidemment pas innocentes, en particulier lorsqu'il s'agit de figurines votives, telles celles de dieux-nains dans le sanctuaire des Cabires, lesquels, à en croire Hérodote (III, 37), épousaient parfois une apparence similaire. Mais les fidèles ne percevaient pas nécessairement les images traditionnelles d'Aphrodite, d'Éros ou d'Athéna qu'ils déposaient dans les Sarapieia comme des manifestations de divinités égyptiennes. Rien ne permet en effet d'envisager systématiquement pour les terres cuites, comme d'ailleurs pour les inscriptions, une telle lecture "syncrétique $»^{17}$ en l'absence d'indices plus explicites. Il parait naturel de rencontrer au sein des sanctuaires déliens des référents aux autres membres du paysage religieux local, d'autant plus lors de la seconde domination athénienne, où la société présentait un caractère très cosmopolite.

9 L'analyse des matériaux, techniques, images et contextes des terres cuites égyptiennes et égyptisantes de Délos amène l'A. à conclure dans son chapitre 6 (Conclusions: Syncretic Theology in Household Cult) que de nombreux habitants de l'île étaient loin d'avoir un intérêt superficiel pour la religion égyptienne. Recouvrant diverses pratiques, notamment votives et domestiques, ces petits artefacts véhiculeraient une théologie nilotique sophistiquée rendue accessible à une audience grecque par leurs concepteurs, suivant peut-être les conseils des prêtres, et reflèteraient l'existence de festivités locales en rapport à ce Nil délien que semble évoquer Callimaque. Les cultes "égyptisants" de Délos auraient ainsi intégré, transformé des traditions authentiquement égyptiennes en y impliquant des individus appartenant à divers milieux culturels. Les négociants y furent particulièrement sensibles, exportant leur dévotion à travers la Méditerranée tout en menant leurs affaires commerciales ${ }^{18}$. Délos apparaît ainsi à l'A. comme un lieu atypique, unique à maints égards, véritable pivot de la diffusion de ces cultes dans le monde romain à une époque de désintégration de la religion civique. Or cette perspective est aujourd'hui largement dépassée, voire totalement invalidée. Bien des dossiers documentaires ont révélé que la polis n'était nullement en déclin à l'époque hellénistique ${ }^{19}$, mais aussi que Délos était seulement un 
relais parmi d'autres ${ }^{20}$ de la propagation de ces cultes qualifiés par l'A. d'« égyptisants». L'usage récurrent de ce label à travers l'étude pose la question de la perception de ces cultes par les Déliens à l'époque tardo-hellénistique, au moment donc où ils utilisaient ces terres cuites. Les concevaient-ils toujours comme "étrangers »? C'est ce qu'invite à croire l'A. en imaginant des stratégies délibérées de la part de fidèles soucieux d'exprimer la compatibilité des traditions religieuses égyptiennes et grecques pour anticiper d'éventuels mouvements de contestation - ce dont il est sérieusement permis de douter ${ }^{21}$. Implantés dès la $1^{\text {re }}$ moitié $\mathrm{du} \mathrm{III}^{\mathrm{e}} \mathrm{s}$. av. J.-C., ces cultes semblent surtout s'être rapidement adaptés aux formes locales, même s'ils conservaient des spécificités, et donc des aspects « égyptisants ", qui ont peut-être fini par nécessiter le recours à des «spécialistes » formés en Égypte, tel cet Hôros du Mont Casion signalé aux côtés du prêtre grec local dans l'enceinte du Sarapieion $\mathrm{A}^{22}$.

Outre les annexes et un recueil de planches de bonne qualité, intégrant également quelques plans et photographies des structures architecturales, l'ouvrage se termine par d'utiles index, dont un consacré aux termes égyptiens, ainsi que par une importante bibliographie où l'on regrettera notamment l'absence de la réflexion terminologique publiée en 2005 par M. Malaise ${ }^{23}$, tout en constatant la présence du RICIS de L. Bricault, auquel ne se réfère toutefois jamais le très riche corps de notes infrapaginales. Si l'analyse menée par l'A. aurait sans doute dû mettre les terres cuites davantage en perspective avec les autres types de monuments isiaques dans le paysage religieux délien, elle a le mérite d'avoir présenté sur le mode anthropologique une documentation souvent négligée dans les études historico-religieuses, en fournissant au lecteur tous les détails nécessaires pour adhérer ou non à la grille de lecture proposée. Ce travail de recherche n'est d'ailleurs pas sans susciter de stimulantes perspectives, révélant notamment la nécessité de constituer un répertoire général du matériel coroplathique isiaque qui mettrait en dialogue les terres cuites déliennes avec celles trouvées sur d'autres sites, comme Amathonte ${ }^{24}$ à Chypre, afin d'affiner notre connaissance des modes de formation, de lecture et d'utilisation de ce type de médias figurés.

\section{NOTES}

1. A. LAUmonieR, Les figurines de terre cuite, Paris, 1956 (EAD, 23) ; A. BOVON, "Chapitre IX. Les figurines ", in Ph. BRUNEAU et al., L'îlot de la Maison des Comédiens, Paris, 1970 (EAD, 27), p. 209-218 ;

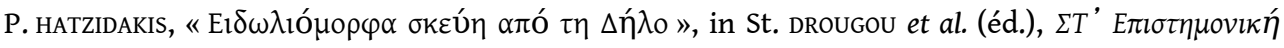

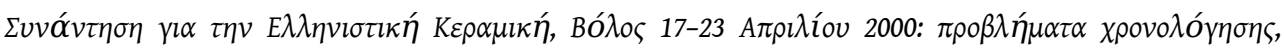

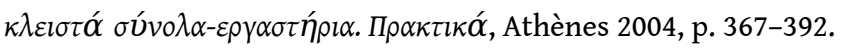

2. Au-delà de la note 3 précisant que le terme désigne des terres cuites fabriquées à l'extérieur de l'Égypte en imitation consciente de figurines égyptiennes.

3. RICIS, no 202/0101.

4. Un qualificatif qui n'est toutefois pas attesté dans les inscriptions pour le Sarapieion B (contra p. 402). 
5. Voir, en attendant la publication par H. Siard du volume de l'EAD consacré aux trois Sarapieia, les études toujours utiles de P. Roussel, Les cultes égyptiens à Délos du IIIe au Ier s. av. J.-C., Paris, 1916, et M.-Fr. BASLEZ, Recherches sur les conditions de pénétration et de diffusion des religions orientales à Délos : IIe-Ier s. avant notre ère, Paris, 1977, p. 35-65.

6. Dont deux inédits inventoriés 59-M-204 (variante du type dit d'«Aphrodite orientale») et F120 (tête d'Isis), ainsi qu'un troisième issu des fouilles de l'Aphrodision (corps drapé avec nœud isiaque).

7. Fr. DUNAND, Religion populaire en Égypte romaine. Les terres cuites isiaques du Musée du Caire, Leyde, 1979 (ÉPRO, 76).

8. Voir les articles réunis dans D.B. THOMPSON, H.A. THOMPSON, Hellenistic Pottery and Terracottas, Princeton, 1987.

9. L'un de ces piliers est ensuite évoqué (p. 332), sans doute erronément, comme « Harpocratic herm ».

10. Décrits par l'A. (p.132-133) comme imitant les "nilomètres » égyptiens, ce qui est techniquement inexact.

11. Fr. DUNAND, s.v. «Agathodaimon », LIMC I (1981), p. 278, no 3-4, comprenant un relief délien, où C.E. Barrett (p. 245-6) propose curieusement de reconnaître Isis et Sarapis encadrant le serpent Agathos Daimon assimilé à Harpocrate. Voir, à ce sujet, les remarques prudentes de M. MALAISE, Pour une terminologie et une analyse des cultes isiaques, Bruxelles, 2005 (Mémoires de la Classe des Lettres de l'Académie royale de Belgique. Collection in-8 ${ }^{\circ}, 3^{e}$ série, 35), p. 162-163.

12. Voir, entre autres, les stimulantes remarques de M.J. VERSLUYS, «Understanding Egypt in Egypt and Beyond », in L. BRICAUlt, M.J. Versluys (éd.), Isis on the Nile. Egyptian Gods in Hellenistic and Roman Egypt. Proceedings of the IVth International Conference of Isis Studies, Liège, November 27-29 2008. M. Malaise in honorem, Leyde/Boston, 2010 (RGRW, 171), p. 12-20.

13. H. SIARD, «L'hydreion du Sarapieion de Délos : la divinisation de l'eau dans un sanctuaire isiaque ", in L. BRICAULT et al. (éd.), Nile into Tiber: Egypt in the Roman World. Proceedings of the IIIrd International Conference of Isis Studies, Leiden, May 11-14 2005, Leyde/Boston, 2007 (RGRW, 159), p. 417-447.

14. M.J. VersLuYs, Aegyptiaca Romana. Nilotic Scenes and the Roman Views of Egypt, Leyde/Boston, $2002(R G R W, 144)$.

15. Ce qui avait conduit P.G.P. меYвоOм, The Nile Mosaic of Palestrina. Early Evidence of Egyptian Religion in Italy, Leyde/New York/Cologne, 1995 (RGRW, 121), p.85-88, à considérer que le répertoire nilotique était parvenu en terre italienne par une autre voie que celle prise par Isis et les siens.

16. C.E. BARRETT, «Harpocrates on Rheneia: Two Egyptian Figurines from the Necropolis of Hellenistic Delos ", in A. MULLER, E. LAFLI (éd.), St. HUYSECOM-HAXHI (coll.), Figurines de terre cuite en Méditerranée grecque et romaine. I: productions et diffusion. II : contextes, iconographie et fonctions (colloque, Izmir, juin 2007), vol. II, Paris/Villeneuve d'Ascq, 2014, sous presse.

17. Qui conduit l'A. à supposer aussi (p.411) que certaines figurines à thèmes totalement helléniques pouvaient être lues en contexte domestique délien sous un mode égyptien.

18. Sur ces réseaux cultuels, voir récemment P. MARTZAVOU, «Les cultes isiaques et les Italiens entre Délos, Thessalonique et l'Eubée », Pallas 84 (2010), p. 181-205.

19. Cf. dernièrement N. DESHOURS, L'été indien de la religion civique. Étude sur les cultes civiques dans le monde égéen à l'époque hellénistique tardive, Bordeaux, 2011 (Scripta Antiqua, 30).

20. Comme Thessalonique (Chr. STEIMLE, Religion im römischen Thessaloniki: Sakraltopographie, Kult und Gesellschaft 168 v. Chr. - 324 n. Chr., Tübingen, 2008 [STAC, 47], p. 79-132 et 184-190) ou Rhodes (Ch. FANTAOUTSAKI, «Preliminary Report on the Excavation of the Sanctuary of Isis in Ancient Rhodes: Identification, Topography and Finds », in L. BRICAULT, R. VEYMIERS [éd.], Bibliotheca Isiaca II, 
Bordeaux, 2011, p. 47-63 [au sein d'un dossier documentaire consacré à divers sites de Grèce, p. 9-129]).

21. C'est ainsi que l'A. interprète (p.303-304) le sénatus-consulte assurant le maintien du Sarapieion A (RICIS, no 202/0195) qui reflète en fait davantage un conflit socio-politique que religieux au moment où l'île passe à nouveau sous contrôle athénien. Voir, en dernier lieu, P. Martzavou, "Isis" et "Athènes": épigraphie, espace et pouvoir à la basse époque hellénistique ", in L. BRICAULT, M.J. VERsLuYs (éd.), Power, Politics and the Cults of Isis. Proceedings of the Vth International Conference of Isis Studies, Boulogne-sur-Mer, October 13-15, 2011, Leyde/Boston, 2014 (RGRW, 180), p. 163-191.

22. RICIS, no 202-0197.

23. Voir supra, n. 30.

24. Voir le dépôt votif publié par A. QUEYREL, Amathonte. IV. Les figurines hellénistiques de terre cuite, Athènes/Paris, 1988 (Études chypriotes, 10), dont les statuettes isiaques, contemporaines de celles de Délos, ne sont pas évoquées par C.E. Barrett.

\section{AUTEURS}

RICHARD VEYMIERS

UMR 8210 ANHIMA - Université de Liège 